\title{
Sub-wavelength near field imaging techniques at terahertz frequencies
}

Maria Caterina Giordano, Leonardo Viti, Oleg Mitrofanov, Gaetano Scamarcio, Stefan Mastel, et al.

Maria Caterina Giordano, Leonardo Viti, Oleg Mitrofanov, Gaetano Scamarcio, Stefan Mastel, Rainer Hillenbrand, Daniele Ercolani, Lucia Sorba, Miriam S. Vitiello, "Sub-wavelength near field imaging techniques at terahertz frequencies," Proc. SPIE 10540, Quantum Sensing and Nano Electronics and Photonics XV, 105400N (26 January 2018); doi: $10.1117 / 12.2291518$

SPIE. Event: SPIE OPTO, 2018, San Francisco, California, United States 


\title{
Sub-wavelength near field imaging techniques at terahertz frequencies
}

\author{
Maria Caterina Giordano, ${ }^{1}$ Leonardo Viti, ${ }^{1}$ Oleg Mitrofanov, ${ }^{2}$ Gaetano Scamarcio,,${ }^{3,4}$ Stefan Mastel, ${ }^{5}$ \\ Rainer Hillenbrand, ${ }^{5}$ Daniele Ercolani, ${ }^{1}$ Lucia Sorba, ${ }^{1}$ and Miriam S. Vitiello ${ }^{1}$ \\ ${ }^{1}$ NEST, Istituto Nanoscienze - CNR and Scuola Normale Superiore, Piazza San Silvestro 12, Pisa, I- \\ 561273 \\ ${ }^{2}$ University College London, Electronic and Electrical Engineering, London, WC1E 7JE, UK \\ ${ }^{3}$ Dipartimento Interateneo di Fisica, Università degli Studi e Politecnico di Bari, via Amendola 173, \\ I-70126 Bari (Italy).
}

${ }^{4}$ CNR-Istituto di Fotonica e Nanotecnologie, sede di Bari, via Amendola 173, I-70126 Bari (Italy)

${ }^{5}$ CIC nanoGUNE Consolider, 20018 Donostia-San Sebastian, Spain

\begin{abstract}
Near-field imaging techniques at terahertz (THz) frequencies are severely restricted by diffraction. To date, different detection schemes have been developed, based either on sub-wavelength metallic apertures or on sharp metallic tips. However high-resolution $\mathrm{THz}$ imaging, so far, has been relying predominantly on detection techniques that require either an ultrafast laser or a cryogenically-cooled $\mathrm{THz}$ detector, at the expenses of a lack of sensitivity when high resolution levels are needed. Here, we demonstrate two novel near-field THz imaging techniques able to combine strongly sub-wavelength spatial resolution with highly sensitive amplitude and phase detection capability. The first technique exploits an interferometric optical setup based on a THz quantum cascade laser (QCL) and on a near-field probe nanodetector, operating at room temperature. By performing phase-sensitive imaging of $\mathrm{THz}$ intensity patterns we demonstrate the potential of our novel architecture for coherent imaging with sub-wavelength spatial resolution improved up to $17 \mu \mathrm{m}$. The second technique is a detector-less s-SNOM system, exploiting a THz QCL as source and detector simultaneously. This approach enables amplitude- and phase-sensitive imaging by self-mixing interferometry with spatial resolution of 60-70 $\mathrm{nm}$.
\end{abstract}

\section{Introduction}

Near-field imaging techniques with subwavelength resolution at infrared (IR) and terahertz (THz) frequencies have recently shown a huge potential in a variety of fields, ranging from nanophotonics and fundamental light-matter interactions ${ }^{1-5}$ to life science ${ }^{6-8}$ and biochemistry, ${ }^{9}$ where non-invasive detection methods are required. To date, different detection schemes 
have been developed, based either on subwavelength metallic apertures ${ }^{10-12}$ or on sharp metallic tip probes ${ }^{13,14}$ (apertureless), that allow to achieve nanoscale spatial resolution well beyond the diffraction limit.

THz near field microscopy presently finds a broad spectrum of applications that include nanoscale imaging of plasmons $\mathbf{s}^{15,16}$ of emergent bi-dimensional (2D) atomic materials (topological insulators, ${ }^{17}$ phosphorene, ${ }^{3}$ silicene, ${ }^{18}$ and their combined van der Waals heterostructures ${ }^{19}$ ), investigation of novel metamaterials and detection of ultrafast dynamics at the nanoscale. ${ }^{3}$ Furthermore, the resonant interaction with fundamental excitations of solids and molecules can be exploited for characterization of fundamental physical properties such as free carrier distribution ${ }^{20,21}$ and phonons, ${ }^{22}$ plasmon polaritons, as well as for investigation of biological systems. This research is essential for the design of novel THz optical components, ${ }^{23,24}$ e.g. metalenses, ${ }^{25}$ metamaterials and devices, as detectors, plasmonic sensors, novel resonators, as well as for the development of non-invasive biosensing methods. ${ }^{6-8}$

Near field optical microscopy based on subwavelength apertures (a-SNOM) ${ }^{10-12}$ has shown a strong potential in the $\mathrm{THz}$ spectral range for large scale imaging $(100 \mu \mathrm{m}-3 \mathrm{~mm})$ of devices, resonant probes, ${ }^{12}$ metamaterials ${ }^{26}$ and biological tissues $^{6}$. In the conventional a-SNOM, spatial resolution is determined by the aperture size $a$, but the possibility to achieve resolution smaller than $\lambda / 100$, being $\lambda$ the wavelength, is practically limited by the suppression of the evanescent field transmission $T$. This indeed follows the power $\operatorname{law}^{10} T \sim a^{6}$. Such a dramatic reduction of the transmitted intensity can be overcome by employing a THz nanodetector ${ }^{27,28}$ integrated into a sub-wavelength aperture which is capable to collect the evanescent component of the transmitted wave. ${ }^{27}$

Alternatively, the apertureless scattering near field optical microscopy (s-SNOM) ${ }^{13,14,20}$ exploits the capability of a sharp metallic tip to behave as an optical nanoantenna, which strongly enhances the electromagnetic field in proximity of its nanometric apex and induces a strong back-scattering in the far field. Since the intensity of the scattered radiation strongly depends on the physical properties of the sample in contact with the tip, as shown by the finite-dipole model ${ }^{29}$, a near field optical image of the sample can be extracted by measuring the scattered light with sensitive detectors. Furthermore, amplitude and phase contrast information can be obtained using interferometric techniques. ${ }^{22,230-32}$ However, near field detection at THz frequencies is still challenging due to the lack of efficient commercial detectors. Indeed, cryogenically 
cooled bolometric systems ${ }^{13}$ have been up to now a mandatory choice to detect the low intensity $\mathrm{THz}$ signals. Very recently cryogen-free s-SNOM nano-imaging has been demonstrated up to $0.75 \mathrm{THz}$ using Schottky diode components. ${ }^{33}$

Here, we show two novel near-field $\mathrm{THz}$ imaging techniques able to combine strongly sub-wavelength spatial resolution with highly sensitive amplitude and phase detection capability.

The first method is based on a near-field probe nanodetector embedded into a subwavelength metallic aperture. Here, the evanescent $\mathrm{THz}$ intensity transmitted through the aperture can be detected by thermoelectric effect. We conceive a simple and versatile interferometric optical setup focusing the THz radiation emitted by a quantum cascade laser (QCL) on the near field probe device. This optical setup enables coherent THz near field imaging with spatial resolution improved up to $17 \mu \mathrm{m}$ and will be described in Section 1 .

The second technique is a detector-less s-SNOM system, exploiting a THz QCL as source and detector simultaneously. This simple optical approach enables self-detection by exploiting self-mixing interferometry. ${ }^{34,35}$ The THz QCL beam is focused at the apex of an oscillating AFM tip and is also employed for detecting the scattered near field radiation. The reinjection of a small fraction $\left(10^{-4}-10^{-2}\right)$ of the emitted field, that coherently interferes within the QCL cavity, allows to detect the scattered signal from the tip as voltage change $(\Delta \mathrm{V})$ across the THz QCL cavity. In this way, the background signal can be suppressed at the higher harmonics of the oscillation frequency of the tip. This novel s-SNOM approach enables highly sensitive near field amplitude and phase imaging with $60-70 \mathrm{~nm}$ of spatial resolution, and will be described in Section 2.

\section{Interferometric detection with near field probe nanodetectors}

A versatile interferometric optical setup based on a QCL and on a near-field probe nanodevice has been exploited in order to achieve near-field $\mathrm{THz}$ detection at room-temperature. A nanowire detector embedded in a sub-wavelength metallic aperture $^{26}$ enables local and coherent detection of the THz near field signal with spatial resolution of $\sim 17 \mu \mathrm{m}$, set by the aperture size.

The field-effect transistor (FET) nanodevice is fabricated by nano-lithography on an undoped Si substrate, transparent to the $\mathrm{THz}$ radiation. The active core of the device is an epitaxially grown, Se-doped InAs nanowire (NW), ${ }^{27}$ 
integrated in a trapezoidal-shaped aperture (Fig. 1a). The InAs NW was grown by bottom-up chemical beam epitaxy and mechanically transferred onto a $350 \mu \mathrm{m}$ thick Si substrate with a $300 \mathrm{~nm}$ insulating $\mathrm{SiO}_{2}$ layer. The source (S)-drain (D) channel, $800 \mathrm{~nm}$ long, was then lithographically defined (Fig. 1a). The stripe-like D electrode connects the right side of the NW and is aligned with the triangularly shaped $\mathrm{S}$ electrode on the opposite side. S and D electrodes were defined with a 10/100 nm Ti/Au metal sequence and subsequently coated with a $120 \mathrm{~nm}$ thick $\mathrm{SiO}_{2}$ layer to prevent current leakage between the channel and the top gate electrode $(\mathrm{G})$. The latter extends to the central NW channel and has been designed as 10/150 nm thick $\mathrm{Cr} / \mathrm{Au}$ screen on the top of the dielectric layer, with a central asymmetric aperture (lateral size $15 \mu \mathrm{m})$.

The peculiar shape of the top gate electrode $(\mathrm{G})$, characterized by a sub-wavelength aperture, allows to asymmetrically feed the THz radiation into the active channel of the FET, thus inducing a temperature gradient $\Delta T$ along the axis of the semiconductor NW. In this way, a steady-state thermoelectric voltage $\Delta u_{S D}=S_{b} \Delta T$ (where $S_{b}$ is the Seebeck coefficient) arises along the channel. The thermoelectric photovoltage is proportional to the THz electromagnetic intesity coupled through the aperture and the detection of the $\mathrm{THz}$ signal is enabled by the diffusion currents induced by the asymmetric feeding of the radiation which produces a not negligible temperature gradient across the FET channel. Additionally, the responsivity of the nanodetector can be easily tailored by changing the gate voltage applied to the FET ${ }^{27}$ In order to demonstrate the detection capability of the aperture integrated NW device, two coherent $\mathrm{THz}$ beams were respectively focused on the aperture side $\left(\mathrm{I}_{\mathrm{a}}\right)$, and on the back side of the detector $\left(\mathrm{I}_{\mathrm{b}}\right)$, as schematically shown in Fig. 1b. A simple interferometric optical setup has been employed (Fig.1c) to focus the THz radiation emitted by a QCL on the two side of the nanodetector. The $3.4 \mathrm{THz}$ QCL, driven in pulsed mode regime (2\% duty cycle) was collimated with a Picarin lens (L, focal length of $25 \mathrm{~mm}$ ) and directed towards an undoped Si wafer acting as beam splitter (BS). The transmitted beam is focused on the aperture side of the probe with a combination of a flat mirror $\left(\mathrm{m}_{1}\right)$ and a parabolic mirror $\left(\mathrm{p}_{1}\right)$. The reflected beam is sent to an optical delay stage made of two pairs of perpendicular mirrors: $\mathrm{m}_{2} \mathrm{~m}_{3}(\mathrm{fixed})$ and $\mathrm{m}_{4} \mathrm{~m}_{5}$ (moveable). Finally, the beam $\mathrm{I}_{\mathrm{b}}$ is focused on the back side of the device by a second parabolic mirror ( $\left.\mathrm{p}_{2}\right)$. The near-field detector is supported by a manual $z$ stage, that controls the position of the device in the focal plane, and by a motorized $x y$-stage, which allows performing two-dimensional $x y$ scans of the interfering THz beams.

Proc. of SPIE Vol. 10540 105400N-4 
Under this optical configuration, the photovoltage signal $\Delta u_{\mathrm{SD}}$, detected across the FET channel, shows a sinusoidal behaviour as a function of the shift $(s)$ of the optical delay stage (Fig.1d). The periodicity of the signal is about $\lambda_{\mathrm{QCL}} / 2=43.5 \mu \mathrm{m}$, and maxima and minima correspond to constructive and destructive interference between the two THz beams $I_{a}$ and $I_{b}$, respectively. The detected interference fringes show the sensitivity of this system to both amplitude and phase of the THz signal. In this way, the coherent near-field detection of subwavelegth objects in close proximity to the aperture is enabled.

Furthermore, the 2D map of the interfering beams can be acquired by detecting the induced photovoltage $\Delta u_{S D}$ in the $x y$ plane for a fixed position of the delay stage. The photovoltage maps acquired for constructive and destructive interference of the two $\mathrm{THz}$ are shown in Fig. $2 \mathrm{a}$ and $2 \mathrm{~b}$, respectively. Since the photovoltage signal $\Delta u_{S D}$ is directly proportional to the optical intensity $\mathrm{I}_{\text {tot }}$ coupled to the aperture nanodevice, the two maps shown in Fig. 2a-2b correspond to the $\mathrm{THz}$ near field distribution in the $x y$ plane for in-phase and out-of-phase interference, respectively. In the first case, a clear interference maximum is detected near the center of the $2 \mathrm{D}$ scan within a spot that reads $\Delta u_{S D}=6.1 \mu \mathrm{V}$, extends for about $100 \mu \mathrm{m}$ and decays following an almost gaussian distribution law. In the second case (Fig.2b), corresponding to $\Delta s$ $=\lambda_{\mathrm{QCL}} / 4$ the intensity map shows a local minimum at the center of the scan, that asymmetrically extends on the left side $(\mathrm{x}<0)$. At the same time, a local maximum is detected around $x \sim 250 \mu \mathrm{m}$ due to the different phase shift between the interfering waves present in this region with respect to the center. Such an asymmetry can be ascribed to a small tilt angle between the acquisition $(x y)$ plane and the wavefront plane, perpendicular to the propagation direction of the two THz waves. These images demonstrate the possibility of retrieving the relative phase distribution of the two beams, taking advantage of the phase-sensitive detection technique.

Additionally, the spatial resolution of the acquired near-field maps can be evaluated by exploiting a micrometric twin needle probe faced to the aperture nanodevice, as schematically shown in Fig. 2c. To this purpose, we employed two PtIr needles mechanically polished to obtain a tip apex radius smaller than $1 \mu \mathrm{m}$. The needles were fixed at a relative angle of $\sim 60^{\circ}$ and at a relative distance between their tips of $\sim 10 \mu \mathrm{m},{ }^{31}$ by means of a micrometer-controlled stage. The twinneedle was aligned parallel to the $y-z$ plane and positioned in close proximity of the aperture nanodevice (i.e. $x=y=0$ ) with a micrometer stage. We then acquired the near field probe photovoltage $\left(\Delta u_{\mathrm{a}}\right)$ induced under illumination of the probe on 
its front side only $\left(\mathrm{I}_{\mathrm{a}}\right)$. Under this condition, a strong localization of the THz field is detected at the apex of the needles due to plasmon guiding of the subwavelegth needles. The detected THz intensity has a gaussian distribution with a FWHM along the confined y-direction of $17 \mu \mathrm{m}$ (Fig $2 \mathrm{~d}$ ). This novel methodology proves that strongly subwavelength spatial resolutions, determined by the aperture size, can be obtained employing by means of a compact nanosensors integrated in the aperture. The growing field of nanoscale $\mathrm{THz}$ detectors, also exploting novel 2D materials, can improve the sensitivity and spatial resolution of this technique and deliver inexpensive room-temperature sub-wavelength resolution THz imaging technology.

\section{Detector-less THz s-SNOM microscopy}

Deep subwavelength spatial resolution can be achieved at IR and $\mathrm{THz}$ frequencies by confining the electromagnetic radiation at the apex of a nanoscale metallic tip in apertureless s-SNOM. ${ }^{13,14,20}$ High resolution $(10-100 \mathrm{~nm})$ optical images of resonant materials can be obtained by detecting the radiation scattered by the tip, placed in contact to the sample surface. Under this condition both amplitude and phase of the near field signal can be retrieved exploiting interferometric

approaches $^{22,30-32}$ that usually require additional optical components and sensitive detectors. This was a major limitation so far, since highly sensitive, room temperature $\mathrm{THz}$ detectors are missing in this spectral range. Therefore, cryogenically cooled bolometers are generally employed to detect the weak $\mathrm{THz}$ frequency signals.

Here, we show a novel approach that allows to overcome this problem: a detector-less s-SNOM system operating in self-detection (SD) mode. We demonstrate near field imaging with spatial resolution of 60-70 nm, determined by the tip size.

The experimental set-up relies on a THz QCL exploited both as radiation source and as a detector for THz radiation backscattered from the tip. The scattered near field intensity is measured via the self-mixing effect, ${ }^{34,35}$ as a voltage change $(\Delta \mathrm{V})$ across the electrical contacts of the QCL. ${ }^{36,37}$ This effect is based on the re-injection of a small fraction of the emitted field that interferes within the laser cavity in a coherent way. Self-mixing interferometry in QCLs has recently shown a strong potential in different configurations, ranging from near field imaging with sub-wavelength spatial resolution $(1-7$ $\mu \mathrm{m}),{ }^{38}$ to the control of the emission of THz QCLs ${ }^{39}$ and the detection of free carrier distribution in semiconductors. ${ }^{40}$ 
In our optical setup (Fig 3a) the $2.7 \mathrm{THz}$ radiation emitted by a QCL is collected by a parabolic mirror ( $\mathrm{p}_{1}$ ), directed to a couple of flat mirrors ( $\mathrm{m}_{1}$ (moveable with a motorized stage) and $\mathrm{m}_{2}$ (fixed) and finally focused at the apex of a Pt AFM tip by a second parabolic mirror $\left(\mathrm{p}_{2}\right)$. The tip is positioned in close proximity to the surface of the sample and is dithered at $\Omega=20 \mathrm{KHz}$ (i.e. tapping mode operation). The optical setup enables the collection of the radiation scattered from the tip and the focusing on the QCL emission facet, along the same optical path. In this way, self-mixing phenomena occur in the QCL cavity and the optical response of the sample can be retrieved by exploiting the Lang-Kobayashi model. ${ }^{41}$ Under the weak feedback regime, the voltage change $\Delta \mathrm{V}$ across the QCL can be written as a function of the ratio between scattered and incident electric field as:

$$
\Delta V \propto A \cos \left(\omega_{0} \frac{2 D}{c}-\phi\right)
$$

where $A$ and $\phi$ are the amplitude and phase of the ratio between scattered and incident field, respectively, $\omega_{0}$ is the unperturbed laser frequency, and $D$ is the laser-tip distance.

In this configuration, the near field component can be efficiently retrieved from the background scattering signal by demodulating the detected $\Delta V$ signal with a lock-in amplifier. Individual harmonics $\Delta V_{i}$ can be recorded up to the order i $=5$, as a function of the distance change $\Delta D$ between the tip and the laser facet. Since the total voltage change $\Delta \mathrm{V}$ can be decomposed in harmonic components as $\Delta \mathrm{V}=\sum_{i=0}^{+\infty} \Delta V_{i} \cos (i \Omega t)$, the optical amplitude $A_{i}$ and phase $\phi_{\mathrm{i}}$ signals can be retrieved as:

$$
\Delta V_{i} \propto A_{i} \cos \left(\omega_{0} \frac{2 D}{c}-\phi_{i}\right)
$$

An important feature of this set-up is the possibility to change the optical phase in the external cavity of the self-mixing interferometer over a period $2 \pi$, by simply translating the position of the flat mirror $\mathrm{m}_{1}$ by $\lambda / 2 \cong 55.5 \mu \mathrm{m}$. The signals $\left|\Delta \mathrm{V}_{2}\right|$ (black line), $\left|\Delta \mathrm{V}_{3}\right|$ (red line) and $\left|\Delta \mathrm{V}_{4}\right|($ blue line) detected on a gold film are shown in Fig. 3b. As expected, they show a periodic trend in which two oscillations of $\left|\Delta V_{i}\right|$ correspond to a shift of $\lambda / 2$.

The potential of the presented technique in mapping the near field THz intensity with nanoscale resolution is demonstrated in Fig.3c-3e, where the AFM topography and the optical signals $\left|\Delta V_{2}\right|$ and $\left|\Delta V_{4}\right|$ are respectively reported. The topography and optical profiles detected at the step edge between a gold film (thickness $110 \mathrm{~nm}$ ) and the underlying Si substrate prove 
the capability of our $\mathrm{THz}$ imaging system to achieve an in-plane resolution comparable with the employed tip apex sizes $(50-70 \mathrm{~nm}) .^{42}$

The nanoscale resolution and the strong near field sensitivity at the higher harmonics can be exploited to image novel 2D materials, whose properties at the nanoscale are currently under study. As a proof of principle demonstration we show in Fig. 4 the optical scan performed on a thin graphene flake lying on a Si substrate is shown. The thickness of the flake is about $6 \mathrm{~nm}$ as shown by the topography image and line profile (Fig.4a-4b)) and is characterized by two folds running parallel to the graphene/substrate edge. This effect can be attributed to the tape-assisted exfoliation procedure employed to transfer the graphene flake on the substrate. The corresponding optical image of $\left|\Delta \mathrm{V}_{2}\right|,\left|\Delta \mathrm{V}_{3}\right|,\left|\Delta \mathrm{V}_{4}\right|$ and $\left|\Delta \mathrm{V}_{5}\right|$ (Fig.4c-f)) clearly shows an enhancement of the near field signal on the graphene flake with respect to the non-resonant $\mathrm{Si}$ substrate. The detected enhancement highlights the potential of the technique for the investigation of plasmons in $2 \mathrm{D}$ materials, phonon-polaritons resonances in crystals and optical modes in semiconductor heterostructures.

In conclusion, amplitude and phase ${ }^{42}$ imaging capability with nanoscale $(60-70 \mathrm{~nm})$ spatial resolution have been demonstrated at THz frequencies by a detector-less s-SNOM system. The detected near field maps show the strong potential of this technique for the study of fundamental light-matter interaction phenomena in atomically thin van der Waals materials and heterostructures. 


\section{Figures}

(c)
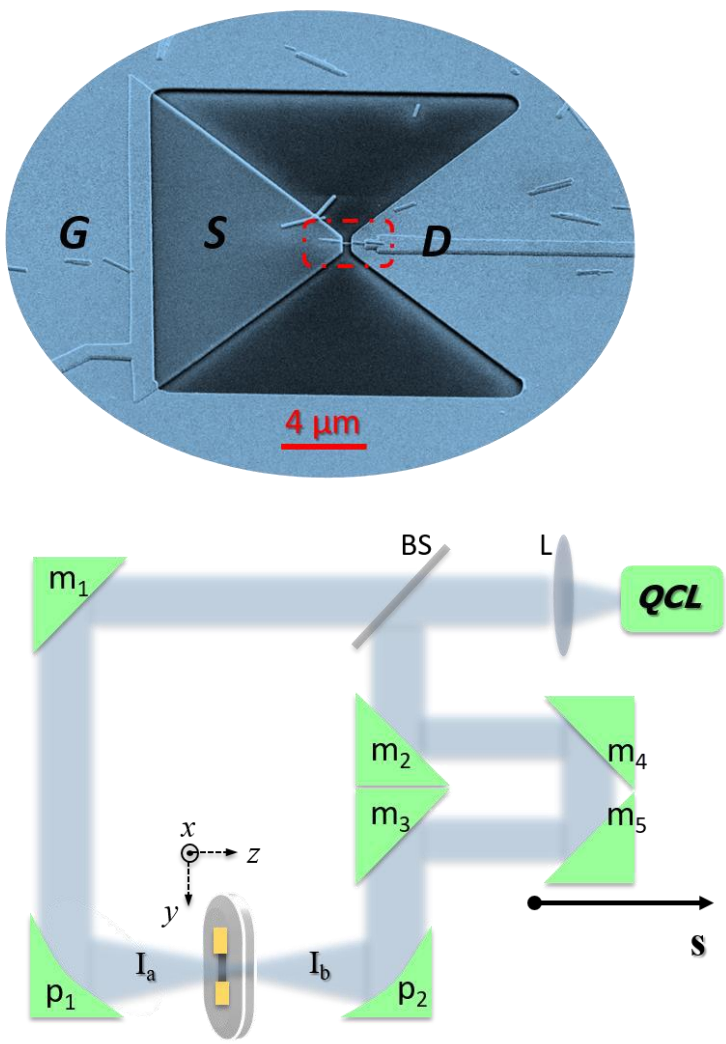

(b)

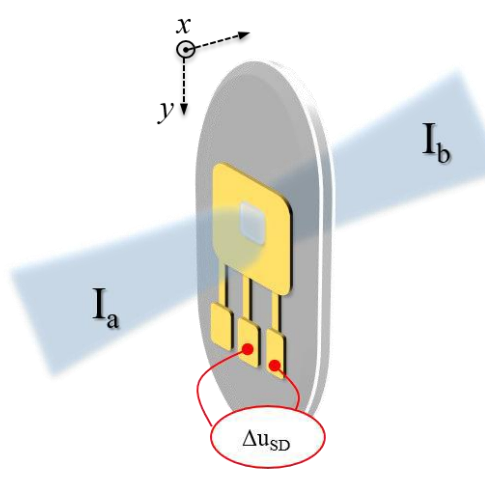

(d)

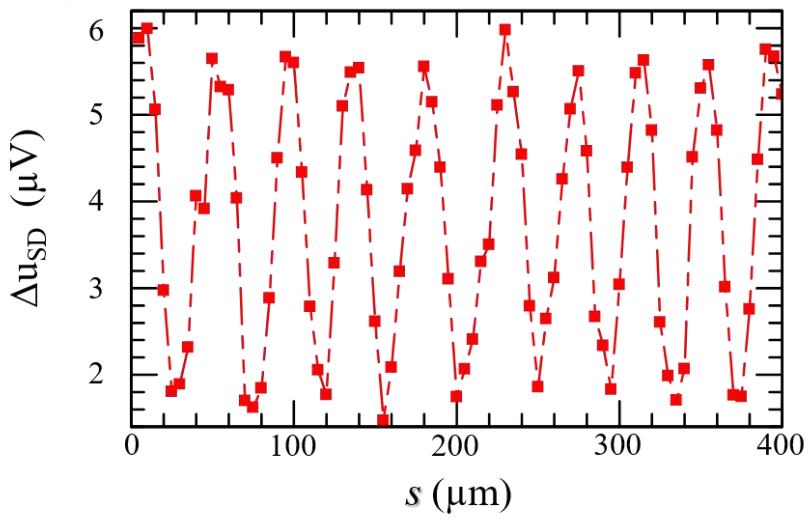

Figure 1: (a) Scanning electron microscopy (SEM) image of the near field THz probe nanodevice (top-view). The red scale bar corresponds to $4 \mu \mathrm{m}$. A top gate contact (G) defines the asymmetric aperture (size: $15 \mu \mathrm{m} \times 15 \mu \mathrm{m}$ ). The central area embeds a InAs nanowire detector with the source (S) drain (D) channel coated with a $\mathrm{SiO}_{2}$ film. (b-c) Schematics of the aperture near-field probe geometry and of the interferometric optical set-up, respectively. (d) Detected photovoltage $\Delta u$ sD under simultaneus THz illumination of the nanodevice on its front $\left(\mathrm{I}_{\mathrm{a}}\right)$ and reference side $\left(\mathrm{I}_{\mathrm{b}}\right)$. The interference fringes are detected as a function of the shift $s$ of the optical delay line. 


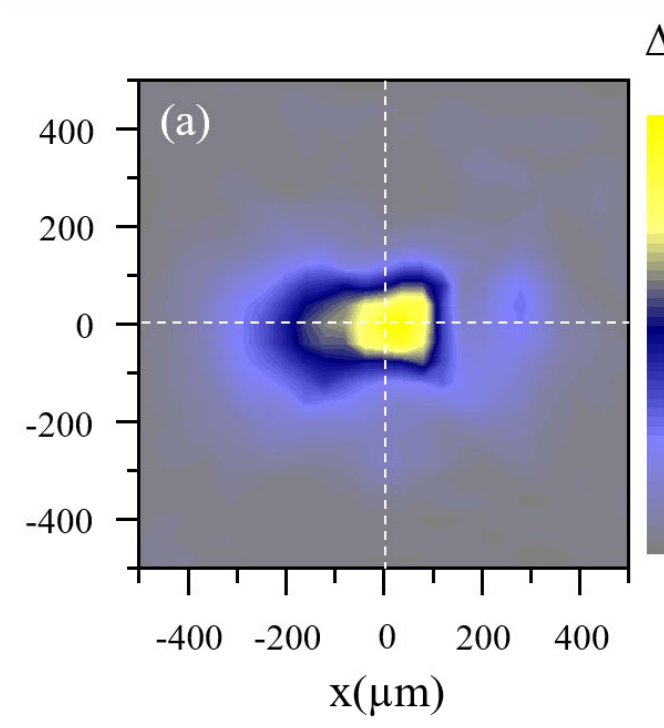

$\Delta u_{\text {in phase }}(\mu \mathrm{V})$
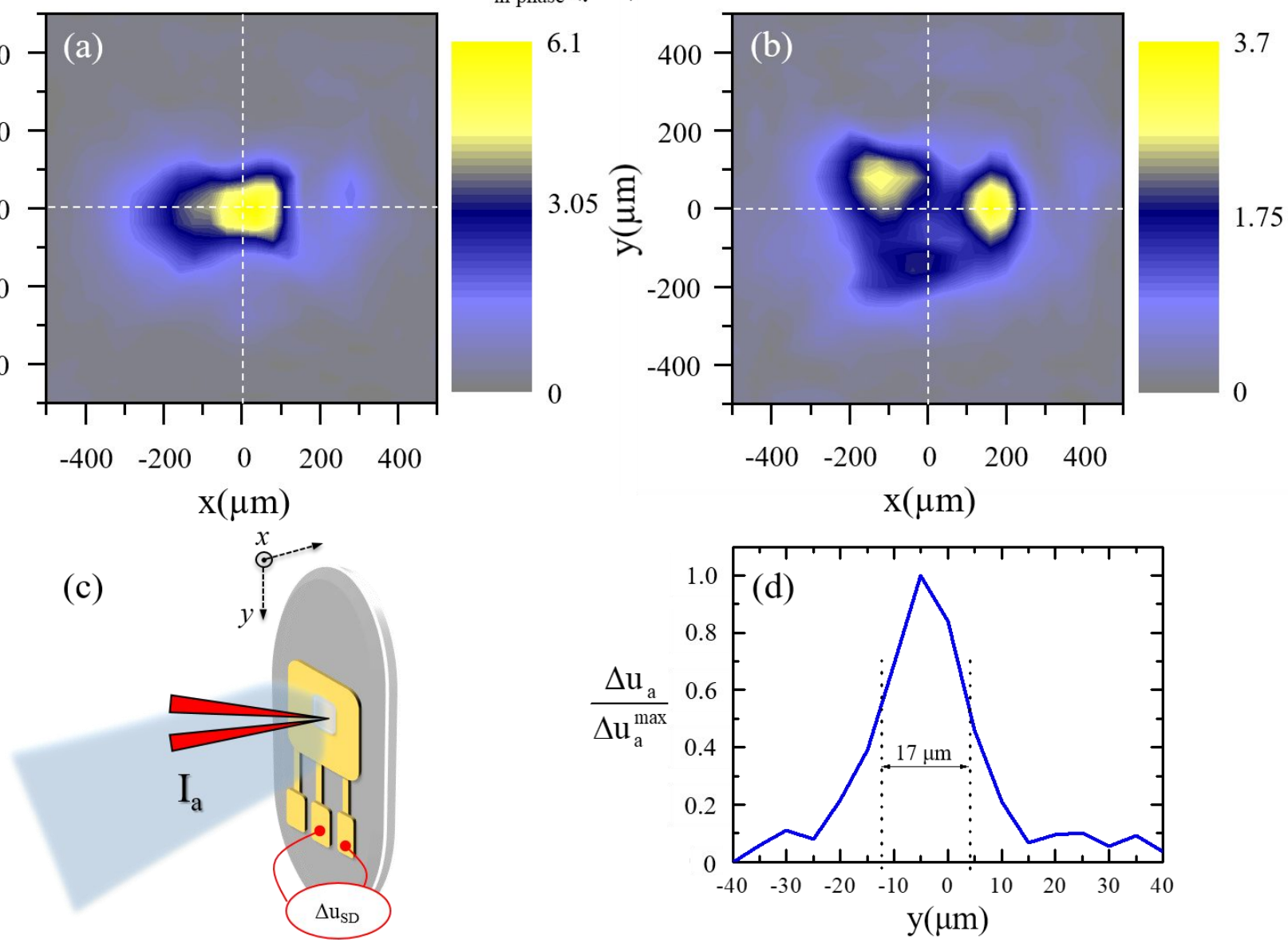

Figure 2: (a,b) Spatial distribution of the near-field probe photovoltage $\Delta u_{S D}$, for in-phase and out of phase interference, respectively. c) Schematics of the twin needle probe facing the NW nano-detector with selective THz illumination on the front side of the nanodetector (Ia). d) Photovoltage $\Delta u_{\mathrm{a}}$ detected at $x=0$ along the $y$-axis. A gaussian profile of the beam is detected with FWHM of $17 \mu \mathrm{m}$. 

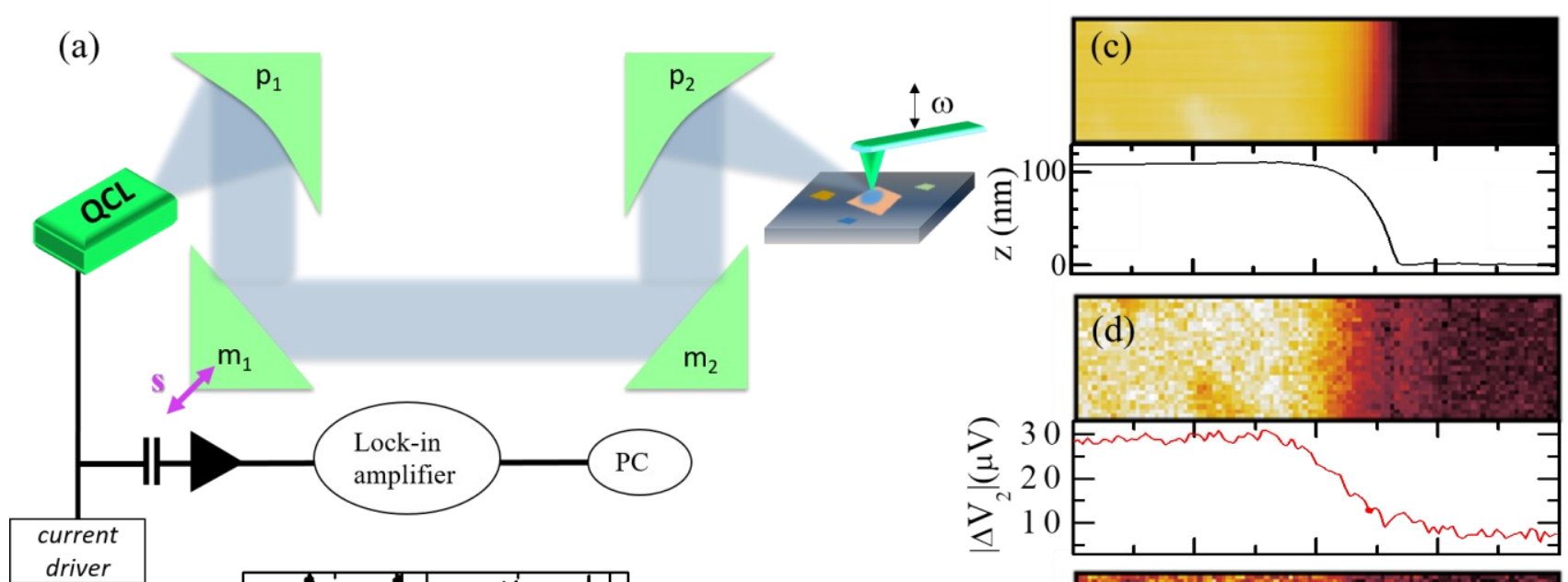

(b)
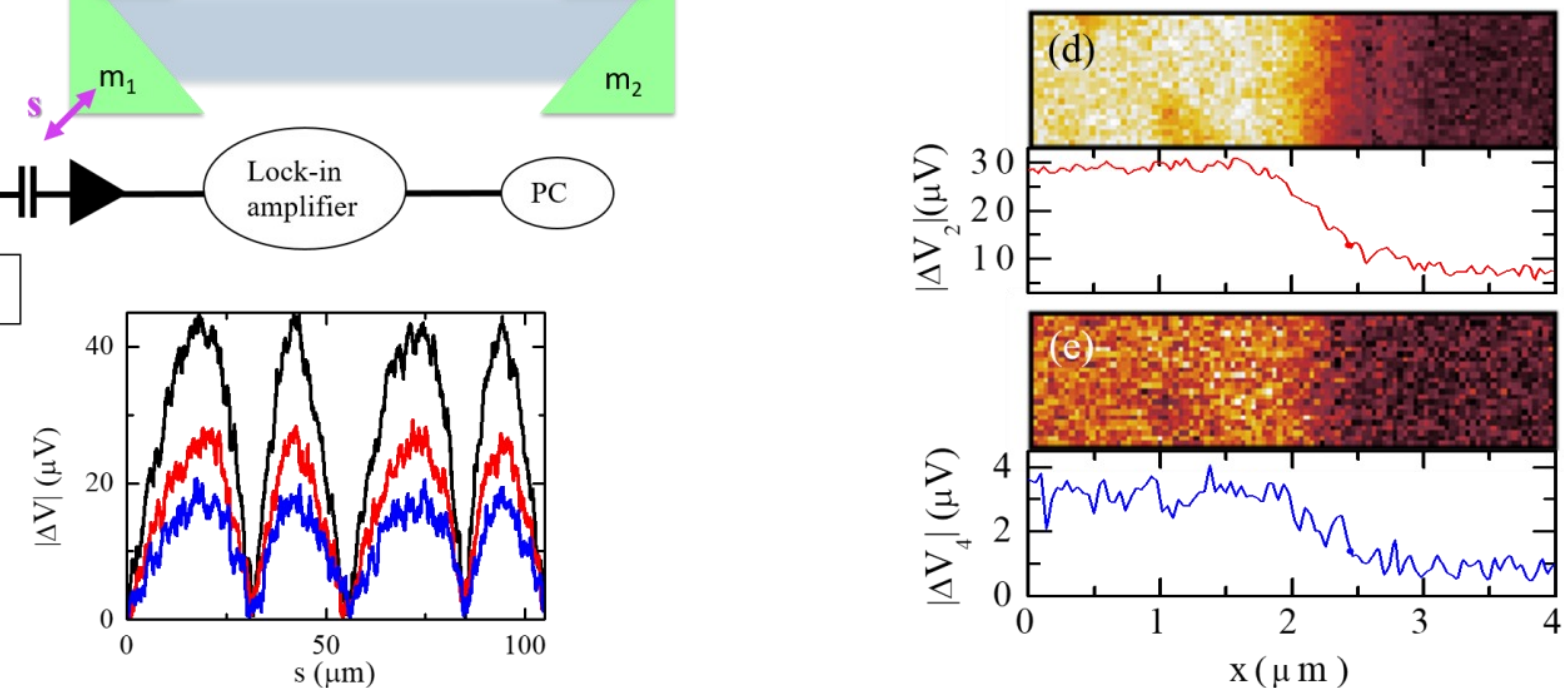

Figure 3: (a) Schematic diagram of the SD s-SNOM system. The following optical components: two parabolic mirrors $\mathrm{p}_{1}$ and $\mathrm{p}_{2}$, one moveable mirror $\left(\mathrm{m}_{1}\right)$ and one fixed mirror $\left(\mathrm{m}_{2}\right)$, focus the THz beam emitted by the QCL onto the apex of a metallic AFM tip dithered at frequency $\Omega$ in close proximity to the sample surface and feeds the scattered radiation back into the QCL cavity to induce self-mixing effects. The distance $D \approx 60 \mathrm{~cm}$ between the QCL front facet and the tip can be progressively varied by shifting the mirror $\mathrm{m}_{1}$ with a piezoelectric motor. (b) Self-mixing-induced modulation of the voltage drop $|\Delta V|$, detected on a gold film surface. The detected signals $\left|\Delta V_{2}\right|$ (black curve) $\left|\Delta V_{3}\right|$ (red curve) and $\left|\Delta V_{4}\right|$ (blue curve) are shown as a function of the shift $s$ of the flat mirror. (c) AFM topography, (d) $2^{\text {nd }}$ harmonic $\left(\left|\Delta \mathrm{V}_{2}\right|\right)$ and (e) $4^{\text {th }}$ harmonic $\left(\left|\Delta \mathrm{V}_{4}\right|\right)$ near-field $\mathrm{THz}$ imaging of the Au-on-silicon sample at a fixed mirror position. 

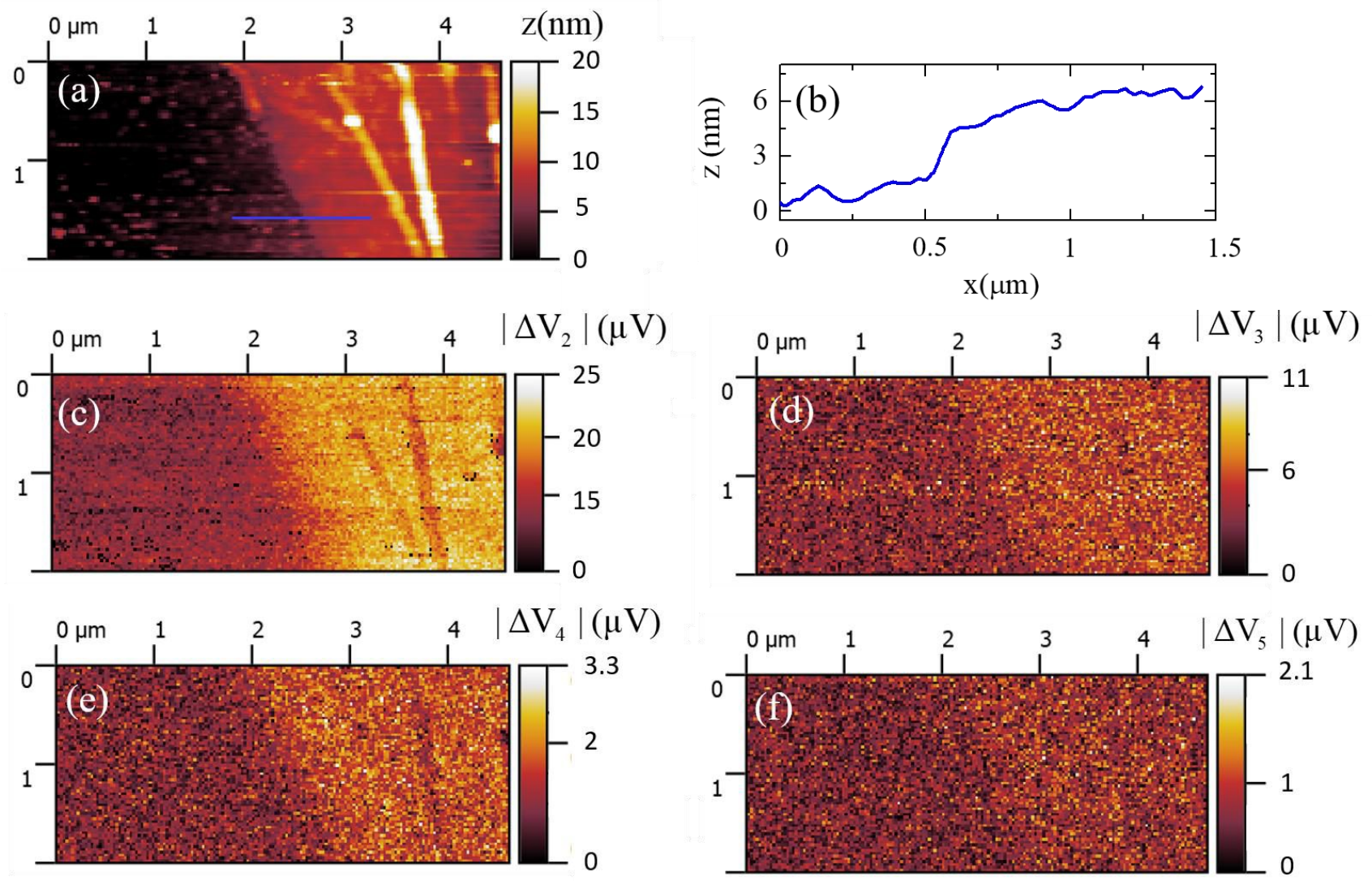

Figure 4: (a,b) AFM topography and (c-f) near-field THz imaging on a 2D graphene flake. The 2D topography map (a) and the line profile (b) at the step edge are compared with the corresponding $\left|\Delta V_{i}\right|$ near field signals $(i=2,3,4,5)$. An enhancement of the near field is detected up to the fifth harmonics on the graphene with respect to the silicon substrate.

\section{Acknowledgements}

The authors acknowledges support from the European Union via the ERC Consolidator Grant SPRINT and the Royal Society. 


\section{References}

[1] Z. Fei, A.S. Rodin, G. O. Andreev, W. Bao, A. S. McLeod, M. Wagner, L. M. Zhang, Z. Zhao, M. Thiemens, G. Dominguez, M. M. Fogler, A. H. Castro Neto, C. N. Lau, F. Keilmann, and D. N. Basov, "Gate-tuning of graphene plasmons revealed by infrared nano-imaging," Nature 487, 7405 (2012).

[2] R. Jacob, S. Winnerl, M. Fehrenbacher, J. Bhattacharyya, H. Schneider, M. T. Wenzel, H. G. von Ribbeck, L. M. Eng, P. Atkinson, O. G. Schmidt, and M. Helm, "Intersublevel Spectroscopy on Single InAs-Quantum Dots by Terahertz NearField Microscopy,” Nano Lett. 12, 4336 (2012).

[3] M. A. Huber, F. Mooshammer, M. Plankl, L. Viti, F. Sandner, L. Z. Kastner, T. Frank, J. Fabian, M. S. Vitiello, T. L. Cocker and R. Huber, "Femtosecond photo-switching of interface polaritons in black phosphorus heterostructures," Nat. Nanotech. 12, 3 (2017).

[4] O. Schubert, M. Hohenleutner, F. Langer, B. Urbanek, C. Lange, U. Huttner, D. Golde, T. Meier, M. Kira, S. W. Koch and R. Huber, "Sub-cycle control of terahertz high-harmonic generation by dynamical Bloch oscillations," Nat. Photon. 8, 2 (2014).

[5] J. Vedran, K. Iwaszczuk, P. H. Nguyen, C. Rathje, G. J. Hornig, H. M. Sharum, J. R. Hoffman, M. R. Freeman, and F. A. Hegmann, "Ultrafast terahertz control of extreme tunnel currents through single atoms on a silicon surface," Nat. Phys. 13, 591 (2017).

[6] H. Chen, W.-J. Lee, H.-Y Huang, C.-M. Chiu, Y.-F.Tsai, T.-F.Tseng, J.-T. Lu, W.-L. Lai and C.-K. Sun, "Performance of THz fiber-scanning near-field microscopy to diagnose breast tumors," Optics Express 19, 19523 (2011).

[7] M.-A. Brun, F. Formanek, A. Yasuda, M. Sekine, N. Ando, and Y. Eishii, "Terahertz imaging applied to cancer diagnosis," Phys. in Med. and Biol. 55, 16 (2010).

[8] L. Rong, T. Latychevskaia, C. Chen, D. Wang, Z. Yu, X. Zhou, Z. Li, H. Huang, Y. Wang, and Z. Zhou, "Terahertz inline digital holography of human hepatocellular carcinoma tissue," Sci. Rep. 5, 8445 (2015).

[9] L.V. Titova, A. K. Ayesheshim, A. Golubov, R. Rodriguez-Juarez, R. Woycicki, F. A. Hegmann, and O. Kovalchuk, "Intense $\mathrm{THz}$ pulses down-regulate genes associated with skin cancer and psoriasis: a new therapeutic avenue?," Sci. Rep. 3, 2363 (2013).

[10] H. Bethe "Theory of diffraction by small holes" Phys. Rev. 66, 163 (1944).

[11] E. Ash and G. Nicholls, "Super-resolution aperture scanning microscope," Nature 237, 510 (1972).

[12] A. J. Macfaden, J. L. Reno, I. Brener and O. Mitrofanov, "3 $\mu \mathrm{m}$ aperture probes for near-field terahertz transmission microscopy,” Appl. Phys. Lett. 104, 011110 (2014).

[13] F. Keilmann and R. Hillenbrand, "Near-field microscopy by elastic light scattering from a tip," Phil. Trans. R. Soc. Lond. A 362, 787-805 (2004).

[14] A. J. L. Adam Review of Near-Field Terahertz Measurement Methods and Their Applications. J. Infrared Milli. Terahz. Waves 32, 976-1019 (2011).

[15] A. Woessner, M. B. Lundeberg, Y. Gao, A. Principi, P. Alonso-González, M. Carrega, K. Watanabe, T. Taniguchi, G. Vignale, M. Polini, J. Hone, R. Hillenbrand and F. H. L. Koppens, "Highly confined low-loss plasmons in grapheneboron nitride heterostructures," Nat. Mater. 14, 421-425 (2015).

[16] P. Alonso-Gonzales, P. et al, Acoustic terahertz graphene plasmons revealed by photocurrent nanoscopy, Nature Nanotechnology, 12, 31-35 (2017). 
[17] M. Autore, H. Engelkamp, F. D’Apuzzo, A. Di Gaspare, P. Di Pietro, I. Lo Vecchio, M. Brahlek, N. Koirala, S. Oh, and S. Lupi, "Observation of Magnetoplasmons in $\mathrm{Bi}_{2} \mathrm{Se}_{3}$ Topological Insulator," ACS Photonics 2, 1231-1235 (2015).

[18] L. Tao, E. Cinquanta, D. Chiappe, C. Grazianetti, M. Fanciulli, M. Dubey, A. Molle and D. Akinwande, "Silicene field-effect transistors operating at room temperature," Nat. Nanotech. 10, 227-231 (2015).

[19] T. Low, A. Chaves, J. D. Caldwell, A. Kumar, N. X. Fang, P. Avouris, T. F. Heinz, F. Guinea, L. Martin-Moreno and F. Koppens, "Polaritons in layered two-dimenisonal materials," Nat. Mater. 16, 182-194 (2017).

[20] A. J. Huber, F. Keilmann, J. Wittborn, J. Aizpurua, and R. Hillenbrand Terahertz Near-Field Nanoscopy of Mobile Carriers in Single Semiconductor Nanodevices, Nano Letters, 8, 3766-3770 (2008)

[21] K.H. Tsai, et al., Looking into Meta-Atoms of Plasmonic Nanowire Metamaterial, Nano Letters 14, 4971-4976 (2014)

[22] R. Hillenbrand, T. Taubner, \& F. Keilmann, Phonon-enhanced light-matter interaction at the nanometer scale. Nature 418, 159-162 (2002).

[23] N. Yu and F. Capasso "Flat optics with designer metasurfaces" Nature Materials 13, 139-150 (2014).

[24] S. H. Lee, J. Choi, H.-D. Kim, H. Choi and B. Min, "Ultrafast refractive index control of a terahertz graphene metamaterial," Scientific Reports 3, 2135 (2013).

[25] S. Zhang et al. "High efficiency near diffraction-limited mid-infrared flat lenses based on metasurface reflectarrays". Optics express, 24(16), 18024-18034, (2016).

[26] D. Denkova, N. Verellen, A.V. Silhanek, V.K. Valev, P.V. Dorpe, \& V.V Moshchalkov, V. V. "Mapping magnetic near-field distributions of plasmonic nanoantennas" ACS nano, 7(4), 3168-3176 (2013).

[27] O. Mitrofanov, L. Viti, E. Dardanis, M. C. Giordano, D. Ercolani, A. Politano, L. Sorba and M. S. Vitiello, "Nearfield terahertz probes with room-temperature nanodetectors for subwavelength resolution imaging," Sci. Rep. 7, 44240 (2017).

[28] L. Viti, J. Hu, D. Coquillat, W. Knap, A. Tredicucci, A. Politano and M. S. Vitiello, "Black Phosphorus Terahertz Photodetectors," Adv. Mater. 27, 5567-5572 (2015).

[29] A. Cvitkovic, N. Ocelic, \& R. Hillenbrand "Analytical model for quantitative prediction of material contrasts in scattering-type near-field optical microscopy”. Optics Express 15, 8550 (2007).

[30] N. Ocelic, R. Hillenbrand, "Subwavelenght-scale tailoring of surface phonon polaritons by focused ion-beam implantation", Nat. Mater. 3, 606-609 (2004).

[31] R. Hillenbrand, F. Keilmann "Complex Optical Constants on a Subwavelength Scale”, Phys. Rev. Lett.,85, 2000, pp. 3029-3032

[32] N. Ocelic, A. Huber, and R. Hillenbrand, "Pseudo-heterodyne Detection for Background- Free Near-Field Spectroscopy”, Appl. Phys. Lett., 89, 2006, 101124

[33] C. Liewald, S.Mastel, J.Hesler, A.J.Huber, R. Hillenbrand, F. Keilmann, “All-electronic THz nanoscopy,” Optica, in press 2018

[34] G. Giuliani, \& S. Donati, Laser interferometry, in Unlocking Dynamical Diversity, John Wiley \& Sons, Ltd, 2005, pp. 217-255.

[35] T. Taimre, M. Nikolić, K. Bertling, Y. L. Lim, T. Bosch, \& A. D., Rakić, "Laser feedback interferometry: a tutorial on the self-mixing effect for coherent sensing", Advances in Optics and Photonics 7, 570-631 (2015).

[36] F.P. Mezzapesa, et al., "Intrinsic stability of quantum cascade lasers against optical feedback" Optics Express 21, 13748 (2013). 
[37] M.S. Vitiello, G. Scalari, B.S. Williams, P. De Natale, "Quantum cascade lasers: twenty years of challenges", Optics Express 23, 8462-8475 (2015).

[38] P. Dean, et al., "Apertureless near-field THz imaging using the self-mixing effect in a quantum cascade laser”, Appl. Phys. Lett. 108, 091113 (2016).

[39] F.P. Mezzapesa, et al., "Photo-generated metamaterials induce modulation of CW terahertz quantum cascade lasers", Sci. Rep. 5, 16207 (2015).

[40] F.P., Mezzapesa, L. Columbo, M. Brambilla, M., Dabbicco, M.S. Vitiello, \& G. Scamarcio, "Imaging of free carriers in semiconductors via optical feedback in terahertz quantum cascade lasers", Appl. Phys. Lett. 104, 041112 (2014).

[41] R. Lang and K. Kobayashi, "External optical feedback effects on semiconductor injection laser properties", IEEE J. Quantum Electron. 16, 347-355 (1980).

[42] M.C. Giordano, S. Mastel, C. Liewald, L. Columbo,M. Brambilla, L. Viti, A. Politano, K. Zhang, L. Li, A. Giles Davies, E.H. Linfield, R. Hillenbrand, F. Keilmann, G. Scamarcio, and M. S. Vitiello "Phase-resolved detector-less terahertz near-field microscopy", Submitted to Science Advances (2017).

[43] T. G. Fiske, and L. B. Coleman, Phys. Rev. B 45, 1414 (1992). 